\title{
Large System Performance of Downlink OFDMA with Limited Feedback
}

\author{
Jieying Chen, Randall A. Berry, and Michael L. Honig \\ Department of Electrical Engineering and Computer Science \\ Northwestern University \\ Evanston, Illinois 60208 \\ Email: j-chenm@northwestern.edu, \{rberry,mh\}@ece.northwestern.edu
}

\begin{abstract}
We consider allocation of sub-channels to users in a downlink OFDMA system. Each user feeds back one bit per sub-channel, which indicates whether or not the gain exceeds a threshold. Users are assigned priority weights, and the thresholds are selected to maximize the weighted sum capacity. We analyze the behavior of the optimal thresholds and growth in capacity, assuming i.i.d. Rayleigh fading sub-channels, in the large system limit in which users $K$ and sub-channels tend to infinity with fixed ratio. If all users have the same priority weight, then the optimized threshold increases as $\log K$ minus a second-order term, which is asymptotically bounded between $\log \log K$ and $\log \log \log K$. Furthermore, the sum capacity per sub-channel increases as $\log \log K$ plus a second-order term, which decreases to a constant as $\log \log K / \log K$. We then consider two classes of users, each assigned a different weight, and show that the capacity of the low priority group tends to zero. Finally, we solve for the optimal thresholds given a fairness constraint on the ratio between the rates of different classes.
\end{abstract}

\section{INTRODUCTION}

Orthogonal Frequency Division Multiple Access (OFDMA) is currently being proposed for emerging wireless cellular and data systems. It has the advantage of eliminating intracell interference by assigning different sets of tones, or subchannels to different users. Furthermore, when combined with opportunistic downlink scheduling [1], the achievable rate region obtained by allowing transmission to multiple users on different sets of sub-channels, which experience frequencyselective fading, is strictly larger than the rate region achieved with time-division multiplexing.

Here we consider downlink OFDMA in a single cell, and study the assignment of sub-channels to users to maximize a weighted sum capacity performance objective. We assume that each sub-channel can be assigned only to a single user. This assignment can be combined with a scheduler, which changes the weights in the sum capacity objective to satisfy fairness constraints.

Given perfect channel state information (CSI) at the transmitter, i.e., knowledge of all users' sub-channel gains, to maximize the sum capacity, the base station would assign a particular sub-channel to the user with the largest corresponding channel gain. Our model is motivated in part by prior work in [5], which considers scheduling for CDMA with a weighted capacity objective given perfect CSI at the transmitter. Other related work on power and rate allocation for downlink OFDMA given perfect CSI at the transmitter is presented in [4] and [7], which maximize an unweighted sum rate performance objective, and in [3], which studies bit loading across sub-channels. Also, in [2] the downlink sum capacity for OFDMA is compared with water-filling and uniform power allocations.

In an OFDMA system with hundreds of sub-channels and many users, full downlink CSI at the transmitter may be difficult to obtain. Namely, hundreds of channel gains must be quantized and relayed to the transmitter during each coherence time, or scheduling interval. To limit the amount of feedback, we assume that the receivers can relay at most one bit per subchannel back to the transmitter. Each bit informs the transmitter whether or not the channel gain falls above an announced threshold, which can differ across users. (In practice, each feedback bit would likely correspond to a coherence band containing many sub-channels.)

We assume that channel gains across users are i.i.d. Rayleigh distributed, and that the users are assigned weights in the sum capacity objective, which reflect priorities or fairness constraints. For each sub-channel there is a set of users with channel gains that exceed their assigned thresholds, and the sub-channel is assigned to the user in that set with the largest weight. (If no user's channel gain exceeds the threshold, then the sub-channel is not assigned, and ties can be settled randomly.) Furthermore, in our model the same power is transmitted on each active sub-channel. This is motivated in part by the results in [4] and [2], which show that optimizing the power spectrum across sub-channels offers only a marginal improvement relative to a uniform power assignment over an optimized subset of sub-channels. Our problem is to find the set of optimal thresholds across users, which maximizes the weighted sum capacity. The weights in the sum capacity objective and the thresholds determine the rates received by the users.

To obtain insight into the behavior of the weighted sum capacity objective and assigned rates as functions of system parameters, we analyze a large system in which the number of users and sub-channels both tend to infinity with fixed ratio. Furthermore, for purposes of analysis we partition the users into groups, corresponding to different priorities, or classes of service. Users within each group are assigned the same weight in the sum capacity objective, and the same threshold.

We start with a single class, and show that the opti- 
mized threshold increases as $\log K$ minus a second-order term, which is asymptotically bounded between $\log \log K$ and $\log \log \log K$. Furthermore, the sum capacity per sub-channel increases as $\Theta(\log \log K)^{1}$ plus a second-order term, which decreases to a constant as $\log \log K / \log K{ }^{2}$ We then consider two classes of users, and show that with optimized thresholds, the capacity of the low priority group tends to zero. Finally, we solve for the optimal thresholds given a fairness constraint on the ratio between the rates of different groups. In that case, the capacity still grows as $\Theta(\log \log K)$, but with a smaller constant than in the unconstrained system.

\section{System Model AND CAPACITY}

The base station transmits data to $K$ users, and the total bandwidth is divided into $N$ i.i.d. sub-channels. Let $h_{k}^{n}$ denote the squared channel gain for the $n^{t h}$ sub-channel of the $k^{t h}$ user, where $1 \leq n \leq N$ and $1 \leq k \leq K$. We assume that the channel gains $h_{k}^{n}, n=1, \cdots, N$, are i.i.d., and are known at receiver $k$. Each user $k$ is assigned a threshold $u_{k}$, and sends back one bit per sub-channel indicating whether or not $h_{k}^{n}$, $n=1, \cdots, N$, exceeds the threshold. ${ }^{3}$

For the $n$th sub-channel let $\mathcal{U}_{n}=\left\{k: h_{k}^{n} \geq u_{k}\right\}$. If $\left|\mathcal{U}_{n}\right|>1$, then the sub-channel is assigned to the user $k \in \mathcal{U}_{n}$ with the largest weight $w_{k}$. The weights therefore represent priorities, and are assumed to be adjusted by a scheduler to achieve a fairness objective. ${ }^{4}$ The rate received by a particular user $k$ then depends on $w_{k}$ and $u_{k}$. Letting $\mathcal{N}_{k}$ denote the set of sub-channels assigned to user $k$, the achievable rate for user $k$, assuming that the transmitter is able to code over a large number of sub-channels, is $R_{k}=$ $\sum_{n \in \mathcal{N}_{k}} E\left(\log \left(1+P h_{k}^{n}\right) \mid h_{k}^{n} \in \mathcal{N}_{k}\right)$, in nats per sub-channel, ${ }^{5}$ where $P$ is the power per sub-channel, the noise variance is normalized to unity, and the expectation is over $h_{k}^{n}$. We assume that the weights are fixed, and that the thresholds $u_{k}$ are selected to maximize the performance objective $C=$ $\sum_{k=1}^{K} w_{k} R_{k}$. We expect that the optimized threshold $u_{k}$ will decrease as the weight $w_{k}$ increases, so that $R_{k}$ increases.

To obtain insight into the behavior of $C$, the optimized thresholds, and the rates $\left\{R_{k}\right\}$, we analyze a large system in which the number of users $K$ and the number of subchannels $N$ both tend to infinity with fixed ratio $\beta=K / N$. Also, the users are partitioned into $M$ groups corresponding to different classes of service. All users in the $m^{\text {th }}$ class, denoted $\mathcal{G}_{m}$, are assigned the same weight $w_{m}$ and the same channel threshold $u_{m}$, and we consider the large system limit in which $K_{m}=\left|\mathcal{G}_{m}\right| \rightarrow \infty$ with fixed ratios $\beta_{m}=K_{m} / N$,

\footnotetext{
${ }^{1}$ We use the notation: $x_{K}=O\left(y_{K}\right)$ if $\lim _{K \rightarrow \infty} \frac{\left|x_{K}\right|}{\left|y_{K}\right|} \leq M ; x_{K}=$ $\Omega\left(y_{K}\right)$ if $y_{K}=O\left(x_{K}\right) ; x_{K}=\Theta\left(y_{K}\right)$ if $x_{K}=O\left(y_{K}\right)$ and $x_{K}=$ $\Omega\left(y_{K}\right) ; x_{K} \asymp y_{K}$ if $\lim _{K \rightarrow \infty} \frac{x_{K}}{y_{K}}=1$.

${ }^{2}$ This is consistent with analogous results for a single-user OFDM channel with partial feedback presented in [6].

${ }^{3}$ The minimum feedback rate for this scheme is the entropy rate of the $N$-bit sequence.

${ }^{4}$ For example, following the utility-based approach to scheduling in [8], [9], we might take $w_{k}=U_{k}{ }^{\prime}(R)$, where $U_{k}(\cdot)$ is a concave increasing function of windowed throughput $R$.

${ }^{5}$ Throughout this paper we assume natural logarithms.
}

$m=1, \cdots, M$. Without loss of generality, we assume that $w_{1}>w_{2}>\cdots>w_{M}$.

The ergodic capacity summed over users in group $m$ is

$$
\begin{aligned}
C_{m}^{K_{m}}= & \frac{1}{N} \sum_{k \in \mathcal{G}_{m}} R_{k}=\operatorname{Pr}\left(\text { sub-channel is assigned to } \mathcal{G}_{m}\right) \\
& \times E\left[\log \left(1+P h_{k}^{n}\right) \mid h_{k}^{n} \geq u_{m} \text { for some } k \in \mathcal{G}_{m}\right]
\end{aligned}
$$

Let $F_{m}(u)=\operatorname{Pr}\left\{h_{k}^{n}<u \mid k \in \mathcal{G}_{m}\right\}$ denote the cumulative distribution function (cdf) of the channel gains for group $m$, and $f_{m}(x)=F_{m}{ }^{\prime}(x)$ denote the associated probability density function (pdf). (We allow different distributions for the different groups.) The probability that a sub-channel is assigned to $\mathcal{G}_{m}$ is therefore $\left(1-g_{m}\right) \prod_{i=1}^{m-1} g_{i}$ where $g_{m}=$ $F_{m}^{K_{m}}\left(u_{m}\right)=\operatorname{Pr}\left\{h_{k}^{n}<u_{m} \forall k \in \mathcal{G}_{m}\right\}$, so that

$C_{m}^{K_{m}}=\left(1-g_{m}\right)\left(\prod_{i=1}^{m-1} g_{i}\right) \int_{u_{m}}^{\infty} \log (1+P x) \frac{f_{m}(x)}{1-F_{m}\left(u_{m}\right)} d x$.

Since the base station allocates constant power $P$ across all active sub-channels, the total transmitted power scales linearly with the number of users. That is, the total power is

$$
K \mathcal{P}=P N\left[1-\prod_{i=1}^{M}\left(1-g_{i}\right)\right]
$$

where $\mathcal{P}$ is the power per user. Given $\mathcal{P}$, we wish to maximize $\sum_{m} w_{m} C_{m}^{K_{m}}$ over the thresholds $u_{1}, \cdots, u_{M}$.

To achieve the rate in (2), each user must compute its own achievable rate, given the assigned set of sub-channels, and relay this rate to the transmitter. That enables the transmitter to select the appropriate codebook for each user. (Another possibility, which requires much more feedback, is for each user to feed back a modulation and coding format for each subchannel, as in [3].) To reduce feedback requirements further, the transmitter could simply compute the rate assuming that $h_{k}^{n}=u_{m}$ for each sub-channel assigned to $\mathcal{G}_{m}$, i.e., $\log (1+$ $\left.P u_{m}\right)$. This requires no additional feedback, but gives a lower ergodic capacity for group $m$, namely,

$$
\underline{C}_{m}^{K_{m}}=\left(1-g_{m}\right)\left(\prod_{i=1}^{m-1} g_{i}\right) \log \left(1+P u_{m}\right)
$$

Proposition 1: Given a set of channel gain cdf's, which satisfy $0<\frac{1-F_{m}(x)}{f_{m}(x)}<\infty$ for all $x>0$ and $1 \leq m \leq M$, as $u_{m} \rightarrow \infty, C_{m}^{K_{m}}=\underline{C}_{m}^{K_{m}}+O\left(\frac{1}{P u_{m}}\right)$.

The proof is based on integrating the expression for $C_{m}^{K_{m}}$ by parts. In the large system limit $(K, N) \rightarrow \infty$, we will see that the optimized threshold $u_{m} \rightarrow \infty$. If $\underline{C}_{m}^{K_{m}} \rightarrow \infty$, then $\underline{C}_{m}^{K_{m}} \asymp C_{m}^{K_{m}}$. In what follows, we will focus on the large system behavior of $\underline{C}_{m}^{K_{m}}$.

\section{Single Class}

We start with a single group of users, i.e., $M=1, K_{1}=$ $K$. We assume that the distribution $F(x)$ is continuous, twice 
differentiable, and that the density $f(x)$ is non-zero for $x>0$. Furthermore, we assume that

$$
\lim _{x \rightarrow \infty} \frac{d}{d x}\left(\frac{1-F(x)}{f(x)}\right)=0 .
$$

From Theorem 2.7.2 in [10], as $K \rightarrow \infty$,

$$
\lim _{K \rightarrow \infty} F^{K}\left(a_{K}+b_{K} x\right) \rightarrow \exp \left(-e^{-x}\right)
$$

uniformly in $x$, where $a_{K}$ and $b_{K}$ satisfy

$$
F\left(a_{K}\right)=1-\frac{1}{K} \quad b_{K}=\frac{1}{K f\left(a_{K}\right)}<\infty .
$$

In other words, the given conditions ensure that with suitable normalization the cdf of the maximum converges to a Gumble cdf. These conditions are satisfied by common fading distributions, such as Rayleigh or Ricean. In what follows, we will assume Rayleigh fading with variance $\sigma^{2}$ for which $a_{K}=\sigma^{2} \log K$ and $b_{K}=\sigma^{2}$.

With some abuse of notation, in this section $u_{K}$ denotes the threshold for a system with $K$ users. Without loss of generality, we can write

$$
u_{K}=a_{K}+b_{K} x_{K}=\sigma^{2}\left(\log K+x_{K}\right),
$$

We consider a sequence of thresholds for which the corresponding sequence $x_{K}$ either converges, or tends to $+/-\infty$. For such sequences, the uniform convergence in (5) implies that

$$
\lim _{K \rightarrow \infty} F^{K}\left(u_{K}\right)= \begin{cases}0, & \text { if } x_{K} \rightarrow-\infty \\ \exp \left(-e^{-x_{0}}\right), & \text { if } x_{K} \rightarrow x_{0} \\ 1, & \text { if } x_{K} \rightarrow \infty\end{cases}
$$

For a sequence of thresholds, we study the asymptotic behavior of the corresponding system capacity,

$$
\underline{C}_{1}^{K}\left(u_{K}\right)=w_{1}\left[1-F^{K}\left(u_{K}\right)\right] \log \left(1+\frac{\beta \mathcal{P} u_{K}}{1-F^{K}\left(u_{K}\right)}\right) .
$$

Proposition 2: As $K \rightarrow \infty$, if $x_{K} \rightarrow-\infty$ and $x_{K}=$ $o(\log K)$, then $\underline{C}_{1}^{K}\left(u_{K}\right) \asymp w_{1} \log (\log K)$. Furthermore, if the sequence $x_{K}$ does not satisfy these assumptions, then $C_{1}^{K}\left(u_{K}\right)=O(\log (\log K))$ with constant strictly less than $w_{1}$.

Proof: Since $x_{K}=o(\log K)$, the threshold $u_{K} \rightarrow \infty$. We therefore have

$$
\begin{aligned}
\underline{C}_{1}^{K} & =w_{1}\left[1-F^{K}\left(u_{K}\right)\right] \log \left(1+\frac{\beta \mathcal{P}}{1-F^{K}\left(u_{K}\right)} u_{K}\right) \\
& \asymp w_{1}\left[1-F^{K}\left(u_{K}\right)\right] \log \left(u_{K}\right)
\end{aligned}
$$

The second asymptotic equivalence follows since $w_{1}[1-$ $\left.F^{K}\left(u_{K}\right)\right] \log \left[\beta \mathcal{P} /\left(1-F^{K}\left(u_{K}\right)\right]\right.$ is bounded when $0 \leq$ $F^{K}\left(u_{K}\right) \leq 1$. Since $x_{K} \rightarrow-\infty$, we have $F^{K}\left(u_{K}\right) \rightarrow 0$ and $\underline{C}_{1}^{K} \asymp w_{1} \log \left(u_{K}\right) \asymp w_{1} \log \log K$.

To show that the capacity cannot grow faster with $K$, we consider the cases $x_{K} \rightarrow x_{0}<\infty$ and $x_{K} \rightarrow \infty$. If $x_{K} \rightarrow x_{0}$, then $F^{K}\left(u_{K}\right) \rightarrow \exp \left(-e^{-x_{0}}\right)$ and

$$
\begin{aligned}
\underline{C}_{1}^{K} & \asymp w_{1}\left[1-\exp \left(-e^{-x_{0}}\right)\right] \log \left(\sigma^{2} \log K+\sigma^{2} x_{0}\right) \\
& \asymp w_{1}\left[1-\exp \left(-e^{-x_{0}}\right)\right] \log \log K .
\end{aligned}
$$

Finally, if $x \rightarrow \infty$, so that $F^{K}\left(u_{K}\right) \rightarrow 1$, following the same approach, we can show that

$$
\underline{C}_{1}^{K} \asymp w_{1} o(\log \log K)
$$

Hence the capacity with a single group and optimized threshold goes to infinity as $\Theta(\log \log K)$, the optimized threshold $u_{K}$ grows as $\Theta(\log K)$, and $F^{K}\left(u_{K}\right) \rightarrow 0$.

The fraction of active channels in a system with $K$ users is denoted as $\eta_{K}\left(u_{K}\right)=1-F^{K}\left(u_{K}\right)$. For a sequence of thresholds as in Proposition 2, $\eta_{K}\left(u_{K}\right) \rightarrow 1$ as $K \rightarrow \infty$.

Lemma 1: Given a sequence of thresholds as in Prop. 2,

Proof: We have

$$
1-\eta_{K}\left(u_{k}\right) \asymp \exp \left(-e^{-x_{K}}\right) .
$$

$$
\begin{aligned}
1-\eta_{K}\left(u_{K}\right) & =F^{K}\left(\sigma^{2} \log (K)+\sigma^{2} x_{K}\right) \\
& =\left(1+\frac{-e^{-x_{K}}}{K}\right)^{K} .
\end{aligned}
$$

Without loss of generality, assume that $x_{K}=-\log \left(w_{K}\right)$, where $w_{K} \rightarrow \infty$. Then

$$
\log \left(\frac{1-\eta_{K}\left(u_{K}\right)}{\exp \left(-e^{-x_{K}}\right)}\right)=K \log \left(1+\frac{-w_{K}}{K}\right)+w_{K} \leq 0
$$

where the last step follows because $K \log \left(1+\frac{x}{K}\right) \leq x$. Hence,

$$
\lim \sup _{K \rightarrow \infty} \frac{1-\eta_{K}\left(u_{K}\right)}{\exp \left(-e^{-x_{K}}\right)} \leq 1
$$

Next note that

$$
\frac{\log \left(1-\eta_{K}\left(u_{K}\right)\right)}{\log \left(\exp \left(-e^{-x_{K}}\right)\right)}=\frac{K}{-w_{K}} \log \left(1+\frac{-w_{K}}{K}\right) \rightarrow 1,
$$

since if $u_{K}$ satisfies Proposition 2, then $w_{K}=o(K)$, and so $\frac{K}{w_{K}} \rightarrow \infty$. Thus, given any $\epsilon$, for $K$ large enough,

$$
\log \left(1-\eta_{K}\left(u_{K}\right)\right) \geq(1-\epsilon) \log \left(\exp \left(-e^{-x_{K}}\right)\right),
$$

and so,

$$
1-\eta_{K}\left(u_{K}\right) \geq\left(\exp \left(-e^{-x_{K}}\right)\right)^{1-\epsilon} .
$$

Dividing both sides by $\left(\exp \left(-e^{-x_{K}}\right)\right)^{1-\epsilon}$ we have

$$
1 \leq \frac{1-\eta_{K}\left(u_{K}\right)}{\left(\exp \left(-e^{-x_{K}}\right)\right)^{1-\epsilon}} \leq\left(\frac{1-\eta_{K}\left(u_{K}\right)}{\exp \left(-e^{-x_{K}}\right)}\right)^{1-\epsilon}
$$

where the last inequality follows since $1-\eta_{K}\left(u_{K}\right) \leq 1$. Therefore,

$$
\lim \inf _{K \rightarrow \infty} \frac{1-\eta_{K}\left(u_{K}\right)}{\exp \left(-e^{-x_{K}}\right)} \geq 1,
$$

and so $1-\eta_{K}\left(u_{K}\right) \asymp \exp \left(-e^{-x_{K}}\right)$, as desired.

Proposition 2 states that the optimal $x_{K}$ 's decrease at a slower rate than $-\log (K)$. From Lemma 1 , if $x_{K}$ increases at a faster rate, then the number of active channels approaches one faster. However, the rate per channel, $\log \left(\log K+x_{K}\right)$ grows at a slower rate. Next we develop tighter bounds on the optimal growth rate of the $x_{K}$-sequence. For this we consider the "remainder" term

$$
\Delta^{K}\left(u_{K}\right)=\underline{C}_{1}^{K}\left(u_{K}\right)-w_{1} \log \left(a_{K}\right) .
$$


This captures the higher order behavior of the total capacity.

Proposition 3: The optimal sequence of thresholds satisfies $x_{K}=O(\log (\log (K)))$ and $x_{K}=\Omega(\log (\log (\log (K))))$ as $K \rightarrow \infty$. With such a sequence,

$$
\Delta^{K}\left(u_{K}\right)-w_{1} \log (\beta \mathcal{P}) \asymp-w_{1} \frac{\log (\log (K))}{\log K} .
$$

In other words, an optimal sequence $x_{K}$ decreases to $-\infty$ no faster than $\log (\log K)$ and no slower than $\log (\log (\log K))$. With such a sequence the remainder term approaches $w_{1} \log (\beta \mathcal{P})$ at the indicated rate. The proof is omitted to save space.

\section{Multiple Classes}

First we consider two groups of users $\mathcal{G}_{1}$ and $\mathcal{G}_{2}$. Continuing to abuse notation, let $u_{K_{m}}$ denote the threshold for $\mathcal{G}_{m}$, and $\mathbf{u}_{K}=\left(u_{K_{1}}, u_{K_{2}}\right)$. The system capacity with $K$ users is given by

$$
\underline{C}_{\text {tot }}^{K}\left(\mathbf{u}_{K}\right)=w_{1} \underline{C}_{1}^{K_{1}}\left(\mathbf{u}_{K}\right)+w_{2} \underline{C}_{2}^{K_{2}}\left(\mathbf{u}_{K}\right),
$$

where $\underline{C}_{m}^{K_{m}}$ is given by (4). Here we assume that users in group $\overline{\mathcal{G}}_{m}$ experience Rayleigh fading with variance $\sigma_{m}^{2}, m=$ 1,2 .

Proposition 4: As $K \rightarrow \infty$, if $x_{K_{1}} \rightarrow-\infty$ and $x_{K_{1}}=$ $o\left(\log K_{1}\right)$, then $\underline{C}_{\text {tot }}^{K}\left(\mathbf{u}_{K}\right) \asymp w_{1} \underline{C}_{1}^{K_{1}}\left(\mathbf{u}_{K}\right) \asymp w_{1} \log \left(\log K_{1}\right)$. Furthermore, if $x_{k}$ does not satisfy these assumptions, then $\underline{C}_{\text {tot }}^{K}\left(\mathbf{u}_{K}\right)=O(\log (\log K))$ with constant strictly less than $w_{1}$.

In other words, with two groups, the higher priority group determines the asymptotic growth rate. The growth rate of the higher priority group is unaffected by the other group, since it receives all channels requested by both groups. From the single-class analysis, the fraction of channels allocated to $\mathcal{G}_{1}$ therefore approaches one.

From the proof of Proposition 4, it follows that with an optimal sequence of thresholds

$$
\lim _{K \rightarrow \infty} \frac{\underline{C}_{2}^{K_{2}}\left(\mathbf{u}_{K}\right)}{\underline{C}_{1}^{K_{1}}\left(\mathbf{u}_{K}\right)}=0,
$$

i.e., the throughput of class 2 users is asymptotically negligible compared to the throughput of class 1 users. The next proposition states that in fact the class 2 throughput asymptotically tends to zero. To see this we consider the remainder term,

$$
\Delta_{\text {tot }}^{K}\left(\mathbf{u}_{K}\right)=\underline{C}_{\text {tot }}^{K}\left(\mathbf{u}_{K}\right)-w_{1} \log \left(a_{K_{1}}\right)
$$

Proposition 5: For the two-class system considered, $\Delta_{\text {tot }}^{K}\left(\mathbf{u}_{K}\right) \rightarrow w_{1} \log (\beta \mathcal{P})$ and $\underline{C}_{2}^{K_{2}}\left(\mathbf{u}_{K}\right) \rightarrow 0$ as $K \rightarrow \infty$.

From the proof of Proposition 5, it follows that an optimal sequence $\left\{x_{K_{2}}\right\}$ satisfies $x_{K_{2}} \rightarrow \infty$ at a rate that satisfies the conditions in Proposition 3; i.e. the thresholds for both groups increase at approximately the same rate. The fraction of channels requested by $\mathcal{G}_{2}$ also converges to one, except that due to the tie-breaking rule, this group gets an arbitrarily small portion of those. Also, we note that to first order, the thresholds for $\mathcal{G}_{2}$ do not effect the growth rate of the total capacity. One conclusion from these results is that in such a system, it may be worth reducing the feedback by having only the users in $\mathcal{G}_{1}$ report their CSI.

This analysis extends to $M>2$ groups. In that case, again asymptotically only the highest priority group has a capacity that is increasing with $K$ at a rate of $w_{1} \log \log (K)$. The capacity of every other group tends to zero. Moreover, it can be shown that for all $m \leq M$, under the optimal thresholds,

$$
\lim _{K \rightarrow \infty} \frac{\underline{C}_{m+1}^{K_{m+1}}}{\underline{C}_{m}^{K_{m}}}=0
$$

i.e., the capacity of class $m+1$ goes to zero faster than the capacity of class $m$.

\section{Multiple Classes with Fixed Rate Ratios}

If instead of using the optimal thresholds derived in the previous section, users in $\mathcal{G}_{1}$ chose $x_{K_{1}}$ to decrease slowly enough so that $\exp \left(-e^{-x_{K_{1}}}\right) \log (\log (K))$ did not converge to zero, then the class 2 users would asymptotically receive a nonzero rate. Moreover, as long as $x_{K_{1}}$ satisfied the conditions in Proposition 2, the total capacity would still be asymptotically equivalent to $w_{1} \log (\log K)$. Alternatively, if $x_{K_{1}} \rightarrow x_{0}$, then both classes would achieve a throughput that increased at rate $\Theta(\log (\log K))$; however, the first-order constant of the total throughput would be strictly less than $w_{1}$. In this section, we study such a variation, where the rates allocated to classes increase asymptotically with a fixed ratio. One reason to consider this modification is to introduce "fairness" among classes. However, we note that if the weights $w_{i}$ dynamically change over time, such modifications may not be needed, e.g., if class weights are inversely proportional to the windowed throughput, as in a proportional fair scheduler [8].

More precisely, we again consider the asymptotic growth rate of $\underline{C}_{\text {tot }}^{K}\left(\mathbf{u}_{K}\right)$ in a system with $M$ groups of users with the additional constraint that as $K \rightarrow \infty$,

$$
\underline{C}_{m}^{K}\left(\mathbf{u}_{K}\right) \asymp \alpha_{m} \underline{C}_{M}^{K}\left(\mathbf{u}_{K}\right),
$$

for $m=1, \ldots, M-1$. The parameters $\alpha_{m}$ are positive constants that specify the asymptotic ratio of each group's (unweighted) capacity to that of $\mathcal{G}_{M}$.

Proposition 6: Given the constraints (12), as $K \rightarrow \infty$ the optimal thresholds for $\mathcal{G}_{M}$ satisfy $x_{K_{M}} \rightarrow \infty$ and $x_{K_{M}}=$ $o(\log K)$. For $m=1, \ldots, M-1$, the optimal thresholds satisfy $x_{K_{m}} \rightarrow x_{m}^{*}$, where

$$
\exp \left(-e^{-x_{m}^{*}}\right)=\frac{1+\sum_{l=m+1}^{M-1} \alpha_{l}}{1+\sum_{l=m}^{M-1} \alpha_{l}} .
$$

With such a sequence of thresholds

$$
\underline{C}_{t o t}^{K} \asymp\left(\sum_{m=1}^{M-1} w_{m} \alpha_{m}+w_{M}\right) \theta^{*} \log (\log K),
$$

where $\theta^{*}=\frac{1}{1+\underset{\substack{M-1 \\ m=1}}{M} \alpha_{m}}$.

Note that

$$
\left(\sum_{m=1}^{M-1} w_{m} \alpha_{m}+w_{M}\right) \theta^{*}<w_{1}\left(\sum_{m=1}^{M-1} \alpha_{m}+1\right) \theta^{*}=w_{1}
$$


Hence the asymptotic growth of $C_{t o t}^{K}$ with the fairness constraints is strictly less than the unconstrained growth. Also, it follows directly from the proof that each class $m$ has a weighted throughput that increases as $w_{m} \alpha_{m} \theta^{*} \log (\log K)$. As an example, suppose that $\alpha_{m}=1$ for each class $m<M$. Then it can be shown that $\theta^{*}=1 / M$, i.e., adding classes asymptotically reduces the capacity of each class as $1 / M$.

\section{NumericAl RESUlTS}

In this section we provide numerical examples, which illustrate our asymptotic results. We assume two classes with weights $w_{1}=1.1$ and $w_{2}=1$. For both groups the channel variance $\sigma_{m}^{2}=1$, and $K_{1}=K_{2}$. Also, we set the ratio $\beta=0.5$, and the power per user $\mathcal{P}=1(0 \mathrm{~dB})$.

Figure 1 shows the weighted capacity per sub-channel for each group, $w_{1} \underline{C}_{1}^{K_{1}}$ and $w_{2} \underline{C}_{2}^{K_{2}}$, and the weighted sum capacity $\underline{C}_{\text {tot }}^{K}$ as a function of the number of users in the system $K$. As expected, $\underline{C}_{\text {tot }}^{K}$ and $w_{1} \underline{C}_{1}^{K_{1}}$ both increase as $\left.\log (\log K)\right)$, and $\underline{C}_{\text {tot }}^{K}$ converges to $w_{1} \log (\log K)+w_{1} \log (\beta \mathcal{P})$, which is also shown in the figure. The capacity for group $2, w_{2} \underline{C}_{2}^{K_{2}}$, decreases very slowly to zero.

Figure 2 shows the optimal thresholds for the two groups, $u_{K_{1}}$ and $u_{K_{2}}$, as a function of $K$. Both tend to infinity no faster than $\log (K)$, and no slower than $\log (K)-\log (\log (K))$, which are also shown.

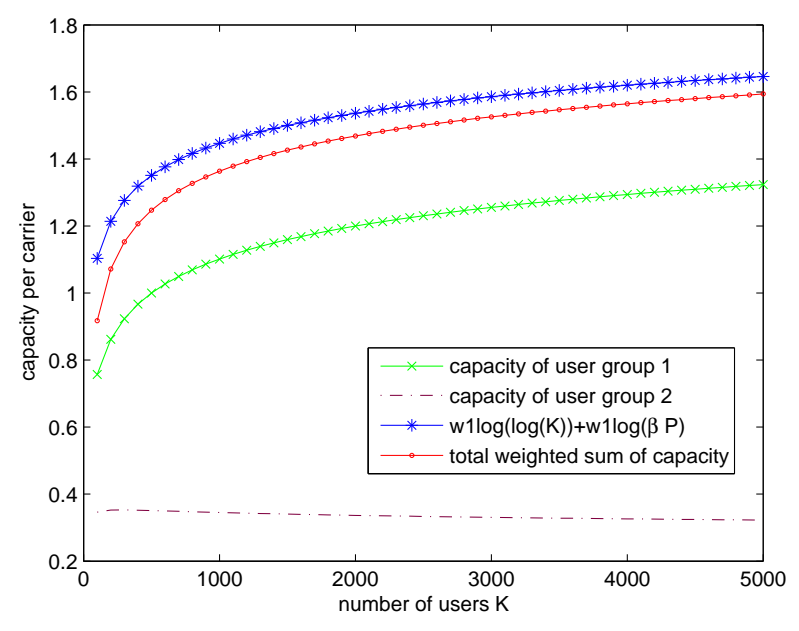

Fig. 1. Rates for two classes vs. total number of users $K$.

\section{CONCLUSION}

We have characterized the asymptotic behavior of a subchannel assignment scheme for downlink OFDMA system. We have assumed that each user feeds back one bit per subchannel, although in practice, the feedback can be reduced further by feeding back one bit for a block of contiguous sub-channels (i.e., within a coherence band). Different quality of service requests are taken into account by assigning the users different priority weights, and by adjusting the feedback thresholds. We have shown that for multiple classes, optimizing the thresholds to maximize the weighted sum capacity

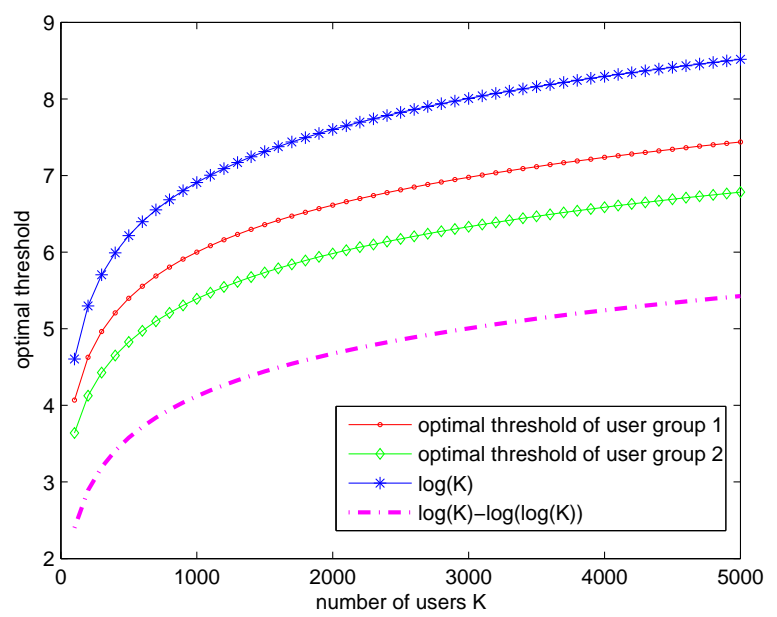

Fig. 2. Optimal thresholds for two classes vs. $K$.

results in the group with highest priority getting all of the subchannels asymptotically. Hence to enforce fairness constraints, either the sum capacity objective must be compromised, as in the fixed rate ratio scenario, or the priorities can be adjusted by a scheduler (or both). Adjusting priorities may enable a reduction in feedback with little loss in spectral efficiency, since only the high priority group would need to report CSI. Although our results were derived for Rayleigh fading channels, we expect that analogous results can be derived for a more general class of fading distributions.

\section{REFERENCES}

[1] X.Liu, E. K. P. Chong, and N. Shroff, "Opportunistic Transmission Scheduling with Resource Sharing Constraints in Wireless Networks," IEEE Journal on Selected Areas in Communications vol.19, no. 10, Oct. 2001.

[2] J. Jang and K. B. Lee, "Transmit Power Adaptation for Multiuser OFDM System," IEEE Journal on Selected Areas in Communications vol.21, no.2 , Feb. 2003.

[3] C. Y. Wong, R. S. Cheng, K. B. Lataief and R. D. Murch, "Multiuser OFDM with Adaptive Subcarrier, Bit and Power Allocation," IEEE Journal on Selected Areas in Communications vol.17, no.10, Oct. 1999.

[4] L. Hoo, B. Halder, J. Tellado, and J. Cioffi, "Multiuser Transmit Optimization for Multicarrier Broadcast Channels: Asymptotic FDMA Capacity Region and Algorithms," IEEE Trans. on Communications, vol. 52, no. 6, June 2004.

[5] R. Agrawal, V. Subramanian, R. Berry, " Joint Scheduling and Resource Allocation in CDMA Systems," Proc. of WiOpt'04, Cambridge, UK, March 2004.

[6] Y. Sun, "Transmitter and Receiver Techniques for Wireless Fading Channels," PhD. Thesis, Northwestern University, 2004.

[7] M. Sharif, "Broadband wireless broadcast channels: throughput, performance, and PAPR reduction," PhD. Thesis, California Institute of Technology, 2005.

[8] R. Agrawal, A. Bedekar, R. La, and V. Subramanian, "A Class and Channel Condition Based Weighted Proportionally Fair Scheduler," Proc. of ITC 2001, Salvidor, Brazil, Sept. 2001.

[9] P. Liu, R. Berry, and M. Honig, "A Fluid Analysis of a Utility-based Wireless Scheduling Policy," to appear IEEE Trans. on Information Theory.

[10] J. Galambos, The Asymptotic Theory of Extreme Order Statistics, John Wiley and Sons, New York, 1978. 